\title{
Hadron modification in a dense baryonic matter
}

\author{
Genis Musulmanbekov ${ }^{1, *}$ \\ ${ }^{1}$ JINR, Dubna, Russia
}

\begin{abstract}
Starting with the Strongly Correlated Quark Model of a hadron structure, SCQM, we demonstrate how the properties of mesons and baryons are modified in a hot and dense nuclear environment. These in-medium modifications can lead to observable effects in heavy ion collisions, such as enhancement of strangeness and dropping vector meson masses.
\end{abstract}

\section{Introduction}

Current and future experiments focus on observables which are sensitive to QGP phase transition, especially to the range of the phase diagram close to the critical point. Observables indicating non-monotonic and unexpected (from theoretical point of view) behavior of emitted particles are especially important. In this way the study of the strange particle production in heavy ion collisions is promising as they could serve a good diagnostic tool to investigate the properties of nuclear matter under extreme conditions. The systematic study of hadron production in central $\mathrm{Pb}+\mathrm{Pb}$ collisions at SPS performed by NA49 collaboration revealed a sharp structure in energy dependence of positive kaon to pion multiplicity ratio, $K^{+} / \pi^{+}[1]$. That peculiarity, called "horn"-effect, was later confirmed by Beam Energy Scan (BES) program of STAR collaboration at RHIC [2] (Fig. 1). At the same time there were no any peculiarities observed in the energetic behaviour of $K^{-} / \pi^{-}$ratio. The idea that strangeness is a good signal of deconfinement was put forward by J. Rafelski in 1982 [3]. The argument was the following: it is energetically favourable to produce

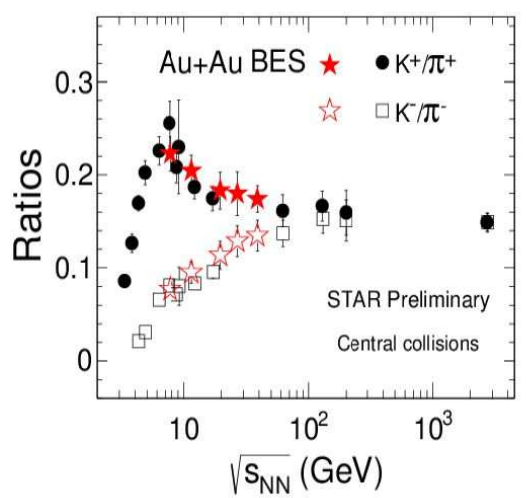

Figure 1. Energy dependence of $K^{ \pm} / \pi^{ \pm}$ ratio for central heavy ion collisions at midrapidity $s \bar{s}$-pairs in deconfined medium than a pairs of strange hadrons in hadron gas. Interpretation of the non-monotonic structure of $\mathrm{K}^{+} / \pi^{+}$has initiated intense theoretical activity. Authors attempted to reproduce the horn structure employing approaches either with phase transition to QGP or without it. "Horn"-like structure has been predicted in Ref. [4], as a manifestation of phase transition between thermalized hadronic and partonic phases. Though a variety of models, statistical [5-8] and kinetic [9, 10] (with or without deconfinement) have been involved to describe "horn" structure, its satisfactory understanding is still not complete.

\footnotetext{
*e-mail: genis@jinr.ru
} 
Another promising observable is a yield of dileptons. Dileptons are an ideal probe to study the properties of hot and dense nuclear matter, since they are emitted at different stages of reaction and leave the medium nearly unperturbed. They allow one a unique access to the properties both of the medium and resonances that decay within a strongly interacting medium. Measurements of emission of dielectrons in different nuclear reactions at wide range of collision energy revealed an enhancement of invariant mass spectra of dileptons yield in the interval $0.2-0.6 \mathrm{GeV}$ [1113] (Fig. 2). This enhancement was interpreted as in-medium modifications of hadrons at high temperature and density resulting in strong broadening of the $\rho$-meson and/or its "mass-dropping" [14-

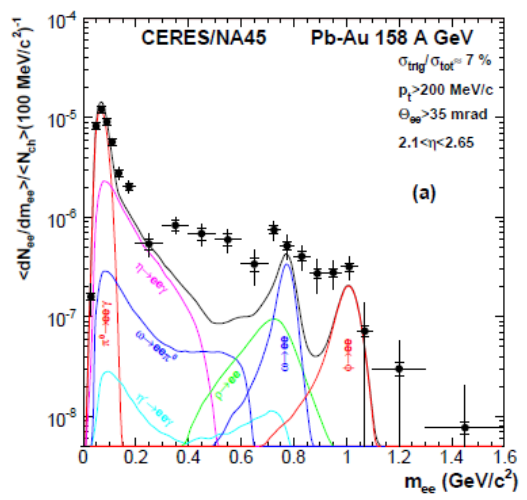

Figure 2. Invariant-mass spectrum of $e^{+} e^{-}$-pairs compared to the expectation from the hadron decay cocktail [12] 16]. We propose our interpretation of the observed phenomena using the Strongly Correlated Quark Model, SCQM, developed by the author [17].

\section{The model}

The real physical vacuum, which energy is below the "empty" perturbative vacuum, is populated by gluon and quark-antiquark condensates. Imagine hypothetically a single quark of a certain color embedded in the physical vacuum. The color field of the quark polarizes the surrounding vacuum that results in production of a condensate. At the same time it experiences the pressure of the vacuum, as a reaction on the ordering, because of the presence of quantum fluctuations of gluon and quark-antiquark fields, or zero point radiation field in a classical sense. Suppose we place a corresponding antiquark in the vicinity of the first quark. Owing to their opposite signs, color polarization fields of the quark and antiquark interfere destructively in the overlapped regions eliminating each other maximally at the middle-point between them. This effect leads to a decreasing value of the condensate density in that region and overbalancing of the isotropic vacuum pressure acting on the quark and antiquark. As a result, an attractive force between the quark and antiquark emerges and the quark and antiquark start to move towards each other. The density of the remaining condensate around the quark (antiquark) is identified with the hadronic matter distribution which is associated with a dynamical mass of the quark. At maximum displacement in the $\bar{q} q$ system corresponding to small overlap of color fields, hadronic matter distributions have maximum extent and densities. The quark (antiquark) in this state possesses a constituent mass. The closer they come to each other, the larger is the destructive interference effect and the smaller hadronic matter distributions around quarks and the larger their kinetic energies. In this state the quark (antiquark) becomes relativistic with a current mass. So, the quark and antiquark start to oscillate around their middle-point. For such interacting $\bar{q} q$ pair located from each other on a distance of $2 x$, the total Hamiltonian is

$$
H=\frac{m_{\bar{q}}}{\left(1-\beta^{2}\right)^{1 / 2}}+\frac{m_{q}}{\left(1-\beta^{2}\right)^{1 / 2}}+V_{\bar{q} q}(2 x),
$$

where $m_{\bar{q}}, m_{q}$ are the current masses of the valence antiquark and quark, $\beta=\beta(x)$ is their velocity depending on displacement $x$, and $V_{\bar{q} q}$ is the quark-antiquark potential energy with 
separation $2 x$. It can be rewritten as

$$
H=\left[\frac{m_{\bar{q}}}{\left(1-\beta^{2}\right)^{1 / 2}}+U(x)\right]+\left[\frac{m_{q}}{\left(1-\beta^{2}\right)^{1 / 2}}+U(x)\right]=H_{\bar{q}}+H_{q},
$$

where $U(x)=\frac{1}{2} V_{\bar{q} q}(2 x)$ is the potential energy of the quark or antiquark. We postulate that the potential energy of quark is equal to its dynamical mass:

$$
2 U(x)=\int_{-\infty}^{\infty} d z^{\prime} \int_{-\infty}^{\infty} d y^{\prime} \int_{-\infty}^{\infty} d x^{\prime} \rho\left(x, \mathbf{r}^{\prime}\right) \approx 2 M_{Q}(x)
$$

with

$$
\rho\left(x, \mathbf{r}^{\prime}\right)=c\left|\varphi\left(x, \mathbf{r}^{\prime}\right)\right|^{2}=c \mid \varphi_{Q}\left(x^{\prime}+x, y^{\prime}, z^{\prime}\right)-\varphi \bar{Q}^{\left.\left(x^{\prime}-x, y^{\prime}, z^{\prime}\right)\right|^{2}}
$$

where $\rho$ is the resulting density of the hadronic matter (quark-antiquark condensate) formed by color fields $\varphi$ and $\varphi_{\bar{Q}}$ of the quark and antiquark, respectively. The structure and shape of vacuum polarization around the color quark/antiquark which could give us the information about the confining potential are not known.

It turned out that our quark-antiquark system behaves similarly to the breather solution of one-dimensional Sine-Gordon equation [18] which in scaled form reads

$$
\square \phi(x, t)+\sin \phi(x, t)=0,
$$

where $\phi(x, t)$ is a scalar function and $x$ and $t$ are dimensionless. It has so-called breather solution

$$
\phi(x, t)_{b r}=4 \arctan \left(\frac{\sqrt{1-w^{2}} \sin (w t)}{w \cosh \left(\sqrt{1-w^{2} x}\right)}\right),
$$

which is the periodic soliton-antisoliton solution for frequencies $w<1$. The energy density profile of the soliton-antisoliton system

$$
\varphi(x, t)_{b r}=d \phi(x, t)_{b r} / d x
$$

behaves the same way as our quark-antiquark system. W. Troost [19] demonstrated that the Hamiltonian (2) corresponded to the breather (soliton-antisoliton) solution of Sine-Gornon equation. He derived the effective potential $U(x)$ for this solution:

$$
U(x)=M \tanh ^{2}(\alpha x)
$$

where $M$ is a mass of soliton/antisoliton and $\alpha$ is an adjusting parameter. Hence, we can identify our potential of quark-antiquark interaction in Hamiltonian (2) with the potential of soliton-antisoliton interaction.

Since quarks are the members of the fundamental color triplet, generalization for the 3-quark system (baryons, composed of Red, Green and Blue quarks) is performed according to $S U(3)_{\text {color }}$ symmetry: a pair of quarks has coupled representations $3 \otimes 3=6 \oplus \overline{3}$ and for quarks within the same baryon only the $\overline{3}$ (antisymmetric) representation is realized. Hence, an antiquark can be replaced by two correspondingly colored quarks to get a color singlet baryon; destructive interference takes place between color fields of three valence quarks (VQs). Putting aside the mass and charge differences of valence quarks one can consider three quarks oscillating synchronously along the bisectors of equilateral triangle turning from the constituent to current state and back. Therefore, the model unifies the features of bag models and constituent models. At a maximal displacement a quark becomes nonrelativistic with a constituent mass corresponding to the maximal value of condensate surrounding it. Further, owing to the prevailing condensate pressure from the outside, it moves 
under influence of the potential (8) (see Fig. 3a) towards two other quarks, and at the origin of oscillation it becomes relativistic with the current mass. Thus, during oscillation quarks transit from constituent states to current states that corresponds to dynamical chiral symmetry breaking and restoration. Important feature of the model is that there is no confining potential/force inside a nucleon. During oscillations (putting aside Coulomb and spin interactions) the interaction force between quarks vanishes both at the origin of oscillation and maximal displacement (Fig. 3b). It becomes maximal in between the origin and maximal displacement. Thus, at the origin of oscillations, quark and antiquark in mesons and three quarks in baryons do not interact, i.e. they are in the state of asymptotic freedom. As to confining potential, it should act at distances exceeding hadronic radii. Apparently, "imprisonment" of quarks is a consequence of the topological nature of hadrons. Hereinafter we assume that the quark-antiquark describing mesons and three quark systems describing baryons are topological solitons. Topological solitons are characterized by the conserving numbers, so-called, winding numbers. For baryons a winding number is identified with the baryonic number. It means that at any temperature and density of nuclear environment baryon conserves its identity and baryonic number. The model meets the local gauge invariance. Indeed, suppose
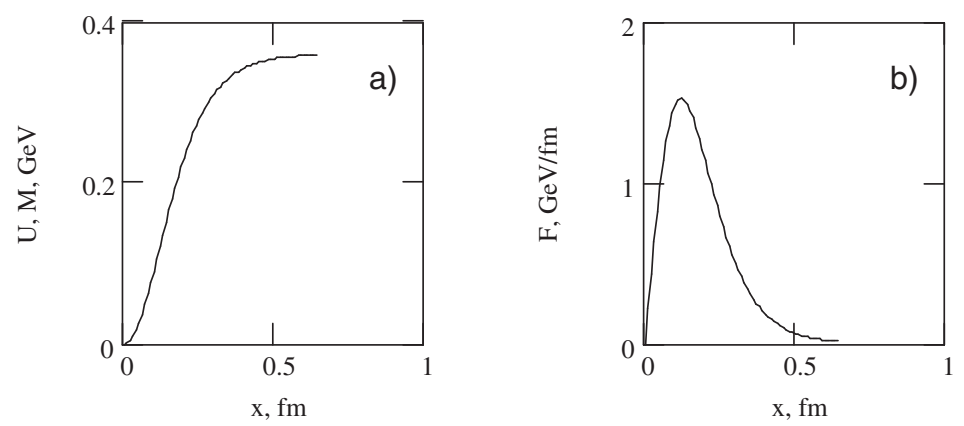

Figure 3. a) Potential energy of a quark and its dynamical (constituent) mass versus its displacement from the origin of oscillation; b) "Confinement" force

$\psi_{\text {color }}$ is a wave function of a single quark in color space where index color accepts one of the values Red, Green, Blue. Interactions of of R, G, and B quarks in a nucleon which result in their oscillations in relation to their center of mass can be reduced to rotation of the wave function phase, $\theta(x)$, of each quark in color space

$$
\psi_{\text {color }}(x) \rightarrow e^{i \theta(x)} \psi_{\text {color }}(x)
$$

This phase rotation results in dressing (undressing) of the quark by quark/qluon condensate that can be linked with transformation of the gauge field $A^{\mu}$ :

$$
A^{\mu}(x) \rightarrow A^{\mu}(x)+\partial^{\mu} \theta(x)
$$

Here we dropped color indices $A_{a}^{\mu}(x) \rightarrow A^{\mu}(x)$ since interactions of color quarks via nonAbelian fields of QCD in our model are reduced to electrodynamical analog

$$
F_{a}^{\mu \nu}=\partial^{\mu} A_{a}^{v}-\partial^{v} A_{a}^{\mu}-\lambda f^{a b c} A_{b}^{\mu} A_{c}^{v} \rightarrow F^{\mu v}=\partial^{\mu} A^{v}-\partial^{v} A^{\mu} .
$$

The parameters of the model are the maximum displacement of valence quark and antiquark in mesons and 3 quarks in baryons, $x_{\max }$, and the parameters of the hadronic matter distribution formed by quark-antiquark condensate around them. In the absence of knowledge about the shape of quark-antiquark condensate around valence quarks, or the form of 
hadronic matter in a constituent quark $\varphi_{Q(\bar{Q})}$, we take it in a Gaussian form:

$$
\varphi_{Q(\bar{Q})}(x, y, z)=\varphi_{Q(\bar{Q})}\left(x_{1}, x_{2}, x_{3}\right)=\frac{(\operatorname{det} \hat{A})^{1 / 2}}{(\pi)^{3 / 2}} \exp \left(-\mathbf{X}^{T} \hat{A} \mathbf{X}\right),
$$

where the exponent is written in a quadratic form.

The value of the maximal quark (antiquark) displacement, and parameters of the Gaussian function for hadronic matter distribution around VQ are chosen to be $x_{\max }=0.64 \mathrm{fm}, \sigma_{x, y}=$ $0.24 \mathrm{fm}, \sigma_{z}=0.12 \mathrm{fm}$. They are adjusted by comparison of calculated and experimental values of the total, inelastic and differential cross sections for $p p$ and $\bar{p} p$ collisions [20]. The mass of the constituent quark at maximum displacement is taken as $M_{Q(\bar{Q})}\left(x_{\max }\right)=$ $\frac{1}{3}\left(\frac{m_{\Delta}+m_{N}}{2}\right) \approx 360 \mathrm{MeV}$, where $m_{\Delta}$ and $m_{N}$ are masses of the delta isobar and nucleon, correspondingly. The current mass of the valence quark is taken to be $5 \mathrm{MeV}$.

\section{Hadron properties in heavy ion collisions}

In head-on collisions of two heavy ion nuclei the energy density in the overlap zone increases drastically. The time of "crossing" two symmetric nuclei through each other when they cease to overlap is $t_{\text {cross }}=2 R / \gamma$, where $R$ is the rest-frame radius of the nucleus. Excited baryons and secondaries in the overlap zone can be considered "formed" at some proper time $\tau_{\text {form }}$ which is $\sim 1 \mathrm{fm} / \mathrm{c}$. At low and moderate collision energies where $\tau_{\text {form }}<t_{\text {cross }}$ particle production and their interactions take place mainly in the overlap zone with high baryonic density. At very high collision energies, once the remnants of Lorenz-contracted disks recede after their initial overlap, the region between them is occupied by a hot and dense "fireball" of interacting secondaries characterized by low baryonic density. The general point of view claims that the hadronic matter at these conditions undergoes the phase transition to quark gluon plasma where quarks become deconfined and the chiral symmetry is restored. Being based on the above model of nucleon structure we offer another scenario. We start with low and intermediate collision energies, when $\tau_{\text {form }}<t_{\text {cross }}$. In the initial stage of collision of nuclei, due to propagation through each other and their Lorenz contraction, the baryonic density in the overlap zone increases more than twice. Correspondingly, the accessible volume occupied by each nucleon composed of light quarks is reduced, at least, more than twice. As a result of accessible volume reduction, the vacuum condensate around the valence quarks decreases that, in turn, leads to reduction of the dynamic mass of quarks and amplitude of oscillation, as shown in Fig. 4. Further compression of nuclear matter could lead to a collapse of nucleons. To avoid collapsing, it is preferable nucleons to transit to delta-isobars and their excited states: $p, n \rightarrow \Delta, \Delta^{*} \ldots$ Parallel alignment of spins of all three quarks leads to their repulsion (according to Pauli principle) that could prevent the collapsing process. Therefore, there should be a limit of accessible volume reduction which can be specified as a "hard-core" of delta isobars and their excited states. However, at higher compression this mechanism is not sufficient because the cores of light quarks need to occupy relatively large volume. Moreover, at higher compression it is preferable for nucleons to be converted to hyperons, as their dimensions/cores are small compared with cores of deltas. This transition of nucleons to hyperons can be described in the framework of ${ }^{0} P_{3}$-model of vacuum. In a compression zone the production of $s \bar{s}$-pairs should be dominating in the content of condensate. $s$-quarks of the pairs replace one or more of $d / u$-quarks of the nucleons, and $\bar{s}$-quarks form together with replaced quarks strange mesons: $p, n \longrightarrow$ hyperons + kaons. In these transition channels the $K^{+} \mathrm{s}$ and $K^{0} \mathrm{~s}$, but any $K^{-}$are produced [21]. At higher compression the production of heavier resonances with all three quark spins aligned parallel should be dominating. However, the transition mechanism works if the "crossing" time, $t_{\text {cross }}$, is larger than formation time, 
$\tau_{\text {form }}$. With increasing collision energy $t_{\text {cross }}$ becomes very short, and the Lorenz-contracted disks with excited baryons fly away leaving behind the hot and dense fireball with a low baryonic chemical potential. Hence, at $\tau_{\text {form }}>t_{\text {cross }}$ the transition mechanism ceases to work. As demonstrated in [21], this mechanism can result in the non-monotonic behavior of the $K^{+} / \pi^{+}$-ratio, the "horn"-effect observed in the experiments. Obviously, this mechanism is additional to the particle production while nuclei propagate through each other. Among produced particles, according to the above arguments, the production of baryon resonances and vector mesons should be dominating.

At essentially high energies the flying away excited remnants of colliding nuclei leave behind them a highly compressed fireball with a low composition of baryons. Its evolution starting with multiparticle production leads to its heating and thermalization and at the last stages to its expansion. Again, since the nuclear matter inside the fireball is highly compressed, the production of pions composed of light $u$ and $d$ quarks in pseudoscalar state is suppressed, and vector mesons, $\rho, \omega, \phi$, and heavier mass resonances will be dominating in the composition of fireball. At the same time there should be essential modification of the features of mesons composed of light quarks $(\rho, \omega)$

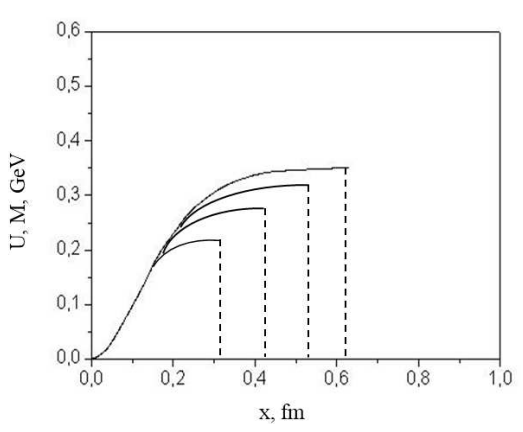

Figure 4. Modification of mass of constituent quark inside compressed nuclear matter

in a compressed medium. Because of reduction of accessible volume and, correspondingly, reduction of condensates around quarks, the masses of these mesons will be depending on the compression value or medium density (Fig. 4). What follows from our model: the more particle density is inside the fireball, the less is the mass of (vector) mesons produced. Without knowing the parameters of fireball we can express this dependence as:

$$
m^{*}=m_{0}\left(1-\alpha \rho / \rho_{0}\right)^{\beta},
$$

where $\alpha$ and $\beta$ are adjustable parameters. In hadronic channels the vector mesons can decay up to the threshold, $2 m_{\pi}$. In dilepton decay mode the threshold continues down to $2 m_{e}$. Therefore, in the framework of our approach, the enhancement of spectral functions of vector mesons (Fig. 2) can be explained by domination of their production and mass dropping. Moreover, the mass dropping effect can be accompanied by the resonance decay width dependence:

$$
\Gamma_{R} \sim \Gamma_{R}^{0}\left(\rho / \rho_{0}\right)^{\gamma}
$$

which results in increasing lifetime of resonances $\tau=1 / \Gamma_{R}^{0}$. Both effects lead to suppression of multiparticle production in a hot and dense medium. During the expansion of fireball, accompanied simultaneously by its cooling, the physical vacuum inside it is restored, that leads to restoration of hadron features.

Analyzing the "horn"-effect and the enhancement of invariant mass spectra of dielectrons in the framework of proposed model, SCQM, we demonstrated that baryons and mesons in a hot and dense medium are essentially modified. 


\section{References}

[1] S. V. Afanasiev et al., Phys.Rev. C 66, 054902 (2002). C. Alt et al., Phys. Rev. C 77, 024903 (2008)

[2] L. Kumar (STAR Collaboration), J. Phys. G:Nucl. Part. Phys. 38, 124145 (2011)

[3] J. Rafelski, Phys. Rep. 88, 331 (1982)

[4] M. Gazdzicki and M. Gorenstein, Acta. Phys. Pol. B 30, 2705 (1999)

[5] J. Cleymans, H. Oeschler, K. Redlich, and S. Wheaton, Phys.Lett. B 615, 50 (2005)

[6] A. Andronic, P. Braun-Munzinger and J. Stachel, Nucl.Phys.A 834, 237C (2010)

[7] K.A. Bugaev et al., arXiv:1412.6571v2 (2014)

[8] S. Chatterjee et al., Phys. Rev. C 81, 044907 (2010)

[9] J.K. Nayak et al., Acta Phys. Slov. 56, 27 (2005)

[10] B. Tomasik and E.E. Kolomeitsev, Eur. Phys. J.C 49, 115 (2007)

[11] G. Agakishiev et al. (HADES Collab.), Phys. Lett. B 663, 43 (2008)

[12] D. Adamova et al. (CERES Collab.), Phys. Rev. Lett. 96, 152301 (2006)

[13] A. Adare et al. (PHENIX Collab.), Phys. Rev. C 81, 034911 (2010)

[14] S. Leupold, V. Metag, and U. Mosel, Int. J. Mod. Phys. E 19, 147 (2010)

[15] R. S. Hayano and T. Hatsuda, Rev. Mod. Phys. 82, 2949 (2010)

[16] E. Bratkovskaya, Nucl. Phys. A 931, 194 (2014)

[17] G. Musulmanbekov in Frontiers of Fundamental Physics (Kluwer Acad./Plenum Pub., New York, 2001) 109-120

[18] R. Rajaraman, Phys. Rep. 21C, 229 (1975)

[19] W. Troost, CERN Report TH.2105-CERN (1975) (unpublished)

[20] G. Musulmanbekov, Phys. Atom. Nucl. 67, 89 (2004)

[21] G. Musulmanbekov, EPJ WoC 138, 03007 (2017) 